ARTICLE

\title{
An ethylene biosynthesis enzyme controls quantitative variation in maize ear length and kernel yield
}

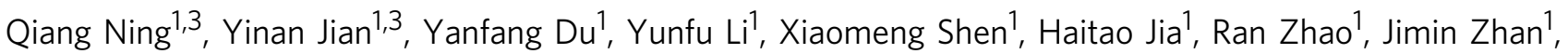
Fang Yang ${ }^{1}$, David Jackson (1) ${ }^{1,2 凶}$, Lei Liu (1) ${ }^{1,2 凶} \&$ Zuxin Zhang (iD) ${ }^{1 凶}$

Maize ear size and kernel number differ among lines, however, little is known about the molecular basis of ear length and its impact on kernel number. Here, we characterize a quantitative trait locus, $q E L 7$, to identify a maize gene controlling ear length, flower number and fertility. qEL7 encodes 1-aminocyclopropane-1- carboxylate oxidase2 (ACO2), a gene that functions in the final step of ethylene biosynthesis and is expressed in specific domains in developing inflorescences. Confirmation of qEL7 by gene editing of ZmACO2 leads to a reduction in ethylene production in developing ears, and promotes meristem and flower development, resulting in a $\sim 13.4 \%$ increase in grain yield per ear in hybrids lines. Our findings suggest that ethylene serves as a key signal in inflorescence development, affecting spikelet number, floral fertility, ear length and kernel number, and also provide a tool to improve grain productivity by optimizing ethylene levels in maize or in other cereals.

\footnotetext{
${ }^{1}$ National Key Laboratory of Crop Genetic Improvement, Hubei Hongshan Laboratory, Huazhong Agricultural University, Wuhan, People's Republic of China.

${ }^{2}$ Cold Spring Harbor Laboratory, Cold Spring Harbor, New York, NY, USA. ${ }^{3}$ These authors contributed equally: Qiang Ning, Yinan Jian.

凶email: jacksond@cshl.edu; lliu@cshl.edu; zuxinzhang@mail.hzau.edu.cn
} 
M aize (Zea mays L.) is the world's most productive cereal crop, partly due to the development of large ears with hundreds of kernels which develop in a stereotypical pattern from fertile florets. The mature female inflorescences of maize usually bear hundreds of kernels, which provide substantial nutrition to meet increasing demands for dietary energy and biofuel consumption by an increasing human population ${ }^{1,2}$. Promoting maize grain yield per unit area by genetic approaches is a sustainable path to double the global supply of maize grains. For high-yield maize breeding, kernel number per ear is one of the key breeding targets ${ }^{3}$. Kernels arise from well-developed and pollinated female florets, borne from spikelet meristems (SMs) derived from the inflorescence meristem (IM) ${ }^{4,5}$. Therefore, IM activity is believed to determine the number of florets and kernels on the maize inflorescence.

A complex functional hierarchy of genes participate in IM maintenance, including the classical CLAVATA-WUSCHEL (CLVWUS) feedback signaling pathway ${ }^{6}$. Phytohormone signaling is also involved in regulating the activity of IMs in maize. Auxin biosynthesis-related genes SPARSE INFLORESCENCE 1 (SPI1) ${ }^{7}$ and VANISHING TASSEL2 $(V T 2)^{8}$, and auxin signaling genes BARREN INFLORESCENCE 1 (BIF1), BIF2 and BIF4,10 influence the initiation of the spikelet paired meristems (SPMs), and mutants in these genes reduce the number of florets on the ear. In addition to auxin, ethylene also regulates many aspects of plant growth and development, including flower development and sex determination ${ }^{11-15}$. In maize, the pleiotropic effect of ethylene was discovered by spraying plants with an appropriate concentration of Ethephon, which is converted to ethylene and is effective as an antilodging agent for small grains. Ethephon can enhance the grain yield when lodging is a problem ${ }^{16}$. In the absence of lodging, low rates of Ethephon can also increase grain yield, but high rates of Ethephon decrease yield ${ }^{16,17}$. Repression of ethylene biosynthesis or response by genetic manipulation enhances maize grain yield under drought and low nitrogen conditions ${ }^{18-20}$, indicating that maize grain yield can be enhanced by modifying ethylene synthesis and signaling. However, the ethylene-related genes that influence grain yield remain largely unknown.

\section{Results and discussion}

$q E L 7$ controls grain yield by affecting floret number and fertility in developing ears. Previously, we identified a major QTL, $q E L 7$, for maize ear length and kernel number and developed a set of QTL near-isogenic lines (NILs) ( $q E L 7^{S L 17}$ and $\left.q E L 7^{Y e 478}\right)^{21}$. $q E L 7^{Y e 478}$ plants had a significant increase in ear length (EL), kernel number per row (KNR), 100-kernel weight and ear weight (EW), and later flowering, compared with $q E L 7^{S L 17}$ (Fig. 1a $-\mathrm{g}$ and Supplementary Table 1), although they were similar in kernel row number, ear diameter, plant height, leaf length and width (Fig. 1d, e, Supplementary Fig. 1a and Supplementary Table 1). Despite having longer ears with more seeds at maturity, developing $q E L 7^{Y e 478}$ ears had significantly shorter ear IMs and fewer florets (Fig. 1h, j, k, Supplementary Fig. $1 \mathrm{~b}$ and Supplementary Table 1). Maize ears are indeterminate, producing florets at the tip that are not fertilized or "filled" to produce seed, and these unproductive or aborted florets are often masculinized. By careful analysis of developing ears, we found that the floret abortion rate of the florets in the $q E L 7^{Y e 478}$ long ear NILs $(\sim 18.7 \%)$ was dramatically lower than in the $q E L 7^{S L 17}$ short ear NILs ( 44.7\%) (Fig. 1i, l, m, Supplementary Fig. 1c and Supplementary Table 1). These results indicate that additional kernels in the long ear $q E L 7^{Y e 478}$ result from a higher proportion of florets that developed into seeds, not from increased floret production.
SNPs and InDels in the $\mathrm{ZmACO2}$ promoter are candidate variants underlying $q E L 7$. To fine map $q E L 7$, we identified seven close recombinants from mapping populations derived from the QTL parents and conducted progeny testing (Supplementary Fig. 1d). By fine mapping, we delineated $q E L 7$ into a 50.8 -kb physical interval flanked by markers M4 and M5 (Fig. 2a, Supplementary Fig. 1d and Supplementary Data 1). Homozygous recombinants carrying the $q E L 7^{Y e 478}$ allele in this interval showed an increase in EL, KNR, and EW, but no change in kernel row number across two environments (Supplementary Fig. 1d and Supplementary Data 1). Only one gene, Zm00001d020686, which encodes 1-aminocyclopropane-1-carboxylate oxidase2 (ACO2), is annotated in this region of B73 RefGenV4 (Fig. 2a), and was referred to as $Z m A C O 2$. Sequencing of $Z m A C O 2$ from the QTL parents revealed five insertion/deletions (InDels) and eleven single nucleotide polymorphisms (SNPs) in its 5'-untranslated region (UTR) and promoter region, but complete sequence identity in the coding region (Fig. 2b). Consistent with these differences in non-coding regions, expression of $\mathrm{ZmACO} 2$ in developing ears differed in the parental lines, with lower expression in the long ear $q E L 7^{Y e 478}$ line than in the short ear $q E L 7^{S L 17}$ line $(P<0.01)$ (Fig. $\left.2 c\right)$. These results suggest that sequence variation in the promoter leads to the differences in $Z m A C O 2$ expression and ear phenotypes between $q E L 7^{Y e 478}$ and $q E L 7^{S L 17}$.

We next asked if sequence variations of $\mathrm{ZmACO} 2$ were associated with ear-traits. We sequenced the 3,539 bp genomic region, covering the promoter and gene body of $\mathrm{ZmACO} 2$, in 214 diverse inbred lines. We identified 56 polymorphic variants (Fig. 2d), and found that four SNPs (-666, -645, -643 and -622) and one $7 \mathrm{bp}$ InDel (-298) in the promoter region were significantly associated with ear length $(p=1.73 \mathrm{E}-04)$ (Supplementary Table 2). Transcription factor (TF) binding motif predictions showed that the $7 \mathrm{bp}$ insertion could introduce new binding sites for bHLH, TCP, and Dehydrin TF families (Supplementary Table 3). The five associated sites, which were in complete linkage disequilibrium, formed two haplotypes: one with $q E L 7^{S L 17}$ genotype and the other with $q E L 7^{Y e 478}$ genotype were named $\mathrm{Hap}^{\mathrm{SL} 17}$ and $\mathrm{Hap}^{\mathrm{Ye} 478}$, respectively (Fig. 2d). Those inbred lines carrying Hap Ye478 exhibited a longer ear with more kernels per row than inbred lines carrying Hap ${ }^{S L 17}$ (Fig. 2e, f), and expression of $\mathrm{ZmACO} 2$ in lines with $\mathrm{Hap} \mathrm{Ye}^{\mathrm{Y}} 7 \mathrm{f8}$ was significantly lower than in the Hap ${ }^{S L 17}$ lines (Fig. 2g). Therefore, we infer that the five associated variants in the $Z m A C O 2$ promoter are possible causal sites that contribute to variations of $Z m A C O 2$ expression and the ear-trait phenotypes. We next sequenced the $Z m A C O 2$ promoter and gene body from 53 teosinte and 44 maize landrace lines to examine the selection pressure acting on this region during maize domestication and improvement (Supplementary Data 3). The promoter region, covering the five associated sites, showed a weak signal of selection, with a considerable reduction in nucleotide diversity $\left(\pi_{\text {maize }} / \pi_{\text {teoisnte }}=0.33\right)$ and a non-neutral evolution pattern $(p=0.02$, HKA test) from teosinte to maize (Supplementary Fig. 2a). However, the favorable $\mathrm{Hap}^{Y e 478}$ haplotype wasn't obviously enriched from teosinte to maize, as it was present in over $50 \%$ of both teosinte and maize lines (Supplementary Fig. 2b), indicating that $\mathrm{Hap}^{\mathrm{Ye} 478}$ emerged before domestication and was maintained at a high frequency during maize domestication and improvement.

ZmACO2 negatively controls ear length and grain yield. To confirm the function of $\mathrm{ZmACO} 2$ in ear development, we created two knockout lines (aco2-cr1 and $c r 2$ ) by CRISPR-Cas9 (Fig. 3a-d and Supplementary Fig. 3a) and three overexpression 

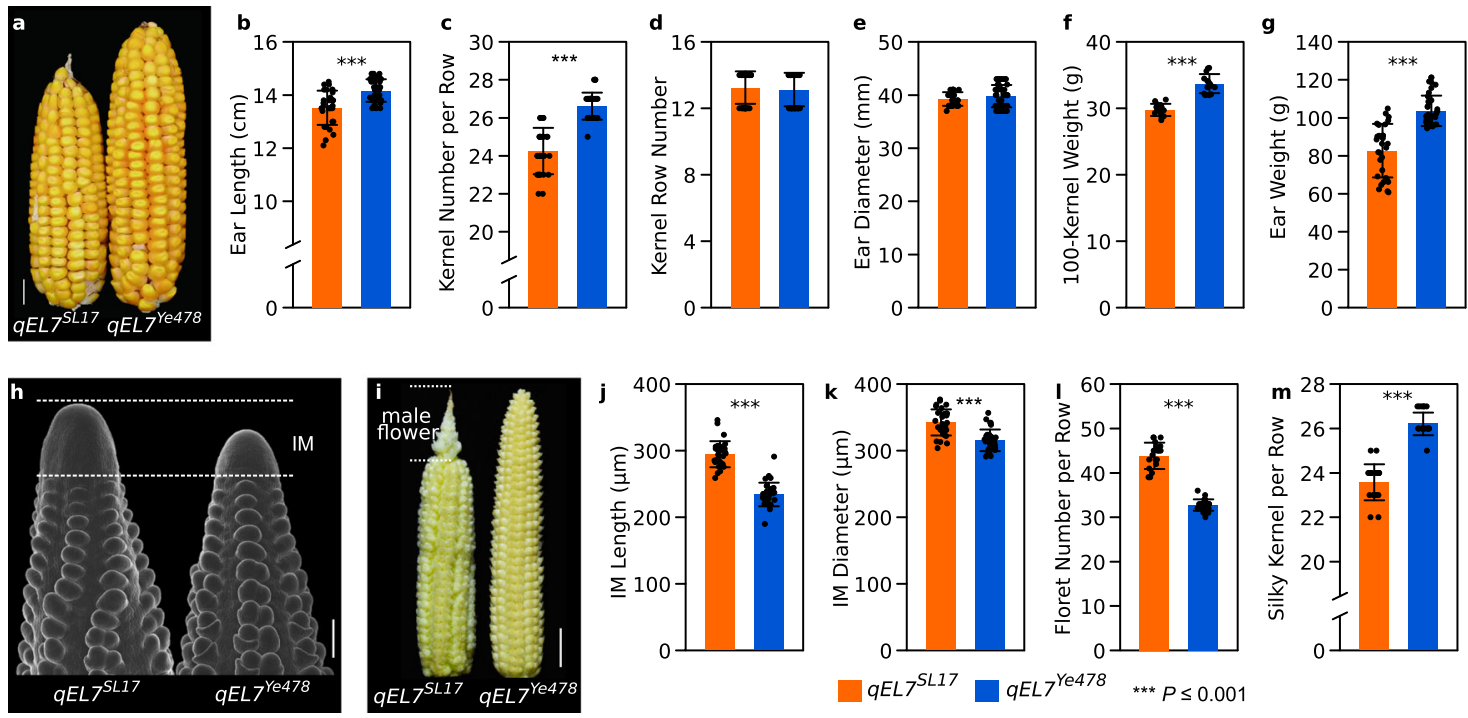

Fig. 1 qEL7 moderates grain yield by affecting the proportion of florets that developed into seeds during ear development. $q E L 7 Y e 478$ plants make longer ear with more kernels per row $(\mathbf{a}$, scale bar $=1 \mathrm{~cm})$, smaller IM (h, scale bar $=200 \mu \mathrm{m})$, and higher floret fertility $(\mathbf{i}$, scare bar $=2 \mathrm{~cm})$ than that in $q E L 7$ SL17. Phenotypes of ear length $\left(\mathbf{b}, p=1.82 \times 10^{-6}\right)$, kernel number per row $\left(\mathbf{c}, p=1.03 \times 10^{-15}\right)$, kernel row number $(\mathbf{d}, p=0.30)$, ear diameter $(\mathbf{e}, p=0.10), 100$-kernel weight $\left(\mathbf{f}, p=7.42 \times 10^{-10}\right)$ and ear weight $\left(\mathbf{g}, p=1.90 \times 10^{-11}\right)$ in $q E L 7^{S L 17}$ and $q E L 7^{Y e 478} ; n=39$ and 32 , respectively. IM length $\left(\mathbf{j}, p=5.92 \times 10^{-19}\right)$ and diameter $\left(\mathbf{k}, p=1.04 \times 10^{-7}\right)$ of $q E L 7 S L 17$ and $q E L 7 Y e 478 ; n=31$ for both. Floret number per row $\left(\mathbf{I}, p=1.24 \times 10^{-20}\right)$ and silky kernels per row $\left(\mathbf{m}, p=9.04 \times 10^{-17}\right)$ of the immature ears before pollination in $q E L 7 S L 17$ and $q E L 7^{Y} e 478 ; n=21$ and 24 , respectively. For $\mathbf{b}-\mathbf{g}$ and $\mathbf{j}-\mathbf{m}$ data are presented as means $\pm \mathrm{SD}$. ${ }^{\star \star \star} p$-value $\leq 0.001$, from a two-tailed, two-sample t-test; orange bar, $q E L 7^{S L 17}$; blue bar, $q E L 7^{Y e 478}$. For $\mathbf{h}$, ears from five plants of each genotype were imaged by scanning electron microscope. $n$ is the number of ears examined in $(\mathbf{b}-\mathbf{g})$ and $(\mathbf{j}-\mathbf{m})$.

lines (ACO2-OE1, OE2, and OE3) (Fig. $3 \mathrm{e}-\mathrm{h}$ ) using maize Ubiquitin promoter-driven $\mathrm{ZmACO} 2$ constructs ( $p U B I: Z m A C O 2$ ). $Z m A C O 2$ loss-of-function lines showed an increase in ear length by $6.8-7.2 \%$ (Fig. 3b), in kernel number per row by $7.9-15.2 \%$ (Fig. 3c), and in ear weight by $13.9-15.1 \%$ (Fig. 3d), compared with the corresponding wild-type siblings. Through the analysis of developing ears, we found aco2-crl plants had a lower floret abortion rate (Supplementary Fig. $3 \mathrm{~b}-\mathrm{g}$ ), consistent with the effect of the long ear allele $q E L 7^{Y e 478}$ in floret fertility (Supplementary Fig. 1c). However, the aco2-cr1 plants also had a larger IM with more florets. These results suggest that aco2 knockout alleles increase kernel number by promoting both floret formation and floret fertility. The aco 2 knockout lines also did not impact other leaf phenotypes, such as senescence (Supplementary Fig. 3h). Overexpressing $Z m A C O 2$ led to a 3-5 fold elevation of transcript levels (Supplementary Fig. 3i), and a decrease in ear length by $9.3-10.9 \%$ (Fig. 3f), in kernel number per row by $8.8-16.0 \%$ (Fig. $3 \mathrm{~g}$ ), and in ear weight by $21.7-25.2 \%$ (Fig. $3 \mathrm{~h}$ ) relative to the wild-type siblings. We confirmed the ear length and kernel number per row changes of overexpression and knockout lines in a second field season (Supplementary Data 4). These results confirmed that $\mathrm{ZmACO} 2$ negatively controls kernel number and grain yield in maize.

In addition to knockout and overexpression of $\mathrm{ZmACO}$, we also mutated its promoter by CRISPR-Cas9 using eight singleguide RNAs (sgRNAs) to target a 520 bp non-repetitive promoter region. The targeted region covered the candidate causal $7 \mathrm{bp}$ InDel (-298) of $q E L 7$, and accessible chromatin detected in developing ears by Assay for Transposase-Accessible Chromatin using sequencing 22 and Micrococcal Nuclease digestion with deep sequencing $^{23}$ (Supplementary Fig. 3j). We identified five promoter-edited alleles ( $A C O 2^{C R}$-pro1 to $\left.A C O 2^{C R-p r o 5}\right)$ with $71-516$ bp deletions in this region (Supplementary Fig. 3k) that decreased $\mathrm{ZmACO} 2$ expression in developing ears (Supplementary Fig. 31). Like aco2-cr knockout alleles, these promoter-edited alleles showed a significant increase in ear length by $6.1-14.7 \%$, in kernel number per row by $8.6-17.8 \%$ and in ear weight by 14.6-24.7\%, compared to wild-type siblings (Supplementary Fig. $3 \mathrm{~m}-\mathrm{o}$ and Supplementary Data 5). Similar changes of these promoter-edited lines in ear length and kernel number per row were observed in a second field season (Supplementary Data 5). Therefore, this promoter region is critical for maintaining $\mathrm{ZmACO} 2$ expression, and its disruption increased ear length and kernel number.

ZmACO2 regulates ethylene biosynthesis in developing ears. $\mathrm{ZmACO} 2$ was expressed widely in roots, internodes, and leaves, with a high expression in inflorescences (Supplementary Fig. 4a). RNA in situ hybridization revealed that $\mathrm{ZmACO} 2$ transcripts were enriched in the adaxial domains of SPMs (Fig. 4a), in semicircular domains at the base of SMs (Fig. 4b), and at the junction between glumes and FMs (Fig. 4c), suggesting a highly localized expression pattern is important for its developmental function. We next investigated the molecular function of ZmACO2. ACC oxidase converts ACC (1-aminocyclopropane-1carboxylate) into ethylene in the final pathway step of ethylene biosynthesis in plants (Fig. 4e) ${ }^{24}$. To confirm the biological activity of $\mathrm{ZmACO} 2$, we purified $\mathrm{ZmACO} 2$ protein and measured its enzyme activity in vitro by quantifying ethylene production at different ACC substrate concentrations. A conserved histidine participates in the binding of a $\mathrm{Fe}^{2+}$ cation required for $\mathrm{ACO}$ catalytic activity ${ }^{25}$, so we made a negative control $\mathrm{ZmACO} 2$ protein with a histidine $(\mathrm{H})$ to glutamine $(\mathrm{Q})$ substitution at this residue $\left(\mathrm{H}_{186} \mathrm{Q}\right)$ (Supplementary Fig. $\left.4 \mathrm{~b}\right)$. The amount of ethylene production catalyzed by $\mathrm{ZmACO} 2$ increased exponentially up to an ACC concentration of $20 \mathrm{mM}$, whereas the negative control $\mathrm{H}_{186} \mathrm{Q}$ proteins had no activity, as expected (Fig. 4f). Next, to ask if $\mathrm{ZmACO} 2$ controlled ethylene biosynthesis in vivo, we measured ethylene levels in dissected developing ears, and found that ethylene levels were significantly lower in the long ear $q E L 7^{Y e 478}$ line than that in $q E L 7^{S L 17}$ (Fig. 4g), which agreed with $Z m A C O 2$ expression in the two NILs (Fig. 2c). We further observed 

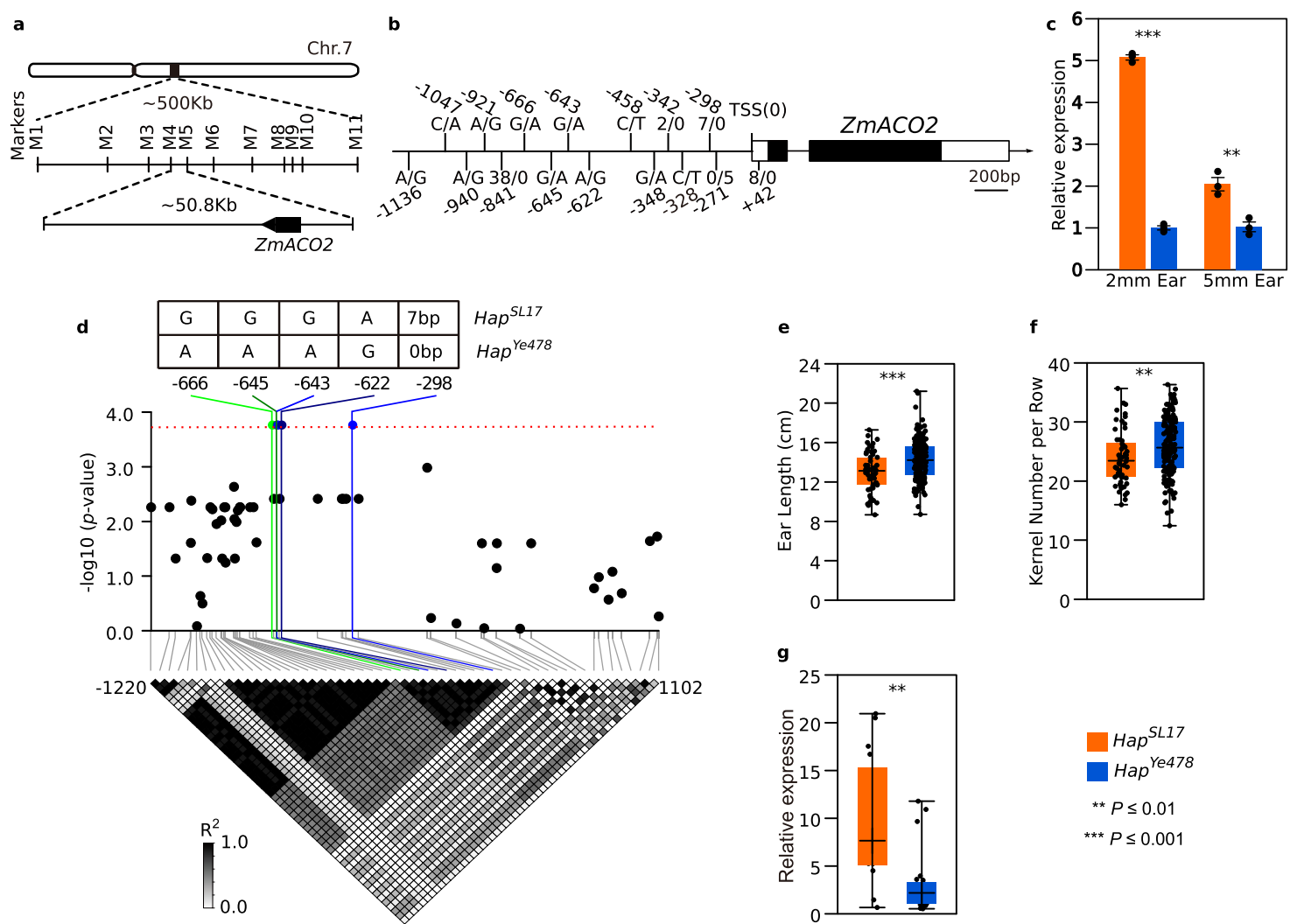

f
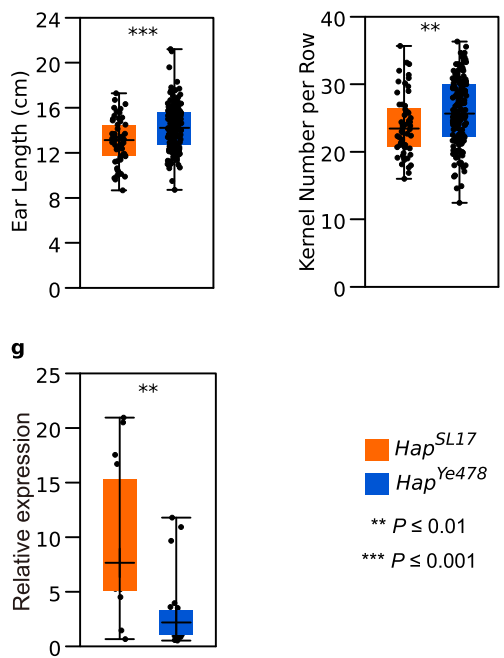

Fig. 2 ZmACO2 is the candidate gene of $\mathbf{q E L 7}$ and negatively affects yield-related traits. a By fine mapping, $q E L 7$ was delineated into a $50.8 \mathrm{~kb}$ interval with only one predicted gene $\mathrm{ZmACO}$; detailed information is presented in Supplementary Fig. 1d. b Sequence variations identified in ZmACO2 genomic regions between NILs; the positions (relative to transcript start site, TSS) were listed beside genotypes information; the genotypes were presented as ${ }_{q E L 7 S L 17 / q E L 7 Y e 478 ;}$ the nucleotide bases and numbers represent the genotypes of SNPs and base pairs of InDels, respectively; white box, UTR region; black box, exon; arrowhead, gene direction. c ZmACO2 expression was lower in the $2 \mathrm{~mm}\left(p=4.33 \times 10^{-7}\right)$ and $5 \mathrm{~mm}(p=0.0034)$ ears of $q E L 7^{Y}$ e 478 (blue bar) than that in $q E L 7 S L 17$ (orange bar), data are presented as means \pm SD and ${ }^{\star \star} p$-value $\leq 0.01,{ }^{\star \star \star} p$-value $\leq 0.001$, from a two-tailed, two-sample t-test. $\mathbf{d}$ Five variants in $\mathrm{ZmACO} 2$ promoter show association with ear length in diversity inbred lines; the red dash line indicates the threshold of significant association at $p \leq 1.79 \times 10^{-4}$; the white to black heatmap shows the pairwise linkage disequilibrium pattern by $R^{2}$; the genotypes and positions (relative to TSS) of five significant association loci are shown on the top and indicated by green and blue dash lines. Hap ${ }^{\text {Ye } 478}$ haplotype has a longer ear $\left(\mathbf{e}, p=6.94 \times 10^{-5}\right)$, more kernel number per row $(\mathbf{f}, p=0.0041)$ and lower $Z m A C O 2$ expression $(\mathbf{g}, p=0.0014, \mathrm{H}=10.26)$ than Hap ${ }^{S L 17}$ haplotype among diverse inbred lines; orange box, Hap ${ }^{S L 77}$ haplotype ( $n=56$ in $\mathbf{e}$ and $\mathbf{f}, n=16$ in $\mathbf{g}$ ); blue box, Hap Ye478 haplotype $(n=158$ in $\mathbf{e}$ and $\mathbf{f}, n=24$ in $\mathbf{g}$ ); each box represents the median and interquartile range, and whiskers extend to maximum and minimum values; ${ }^{\star \star} p$-value $\leq 0.01,{ }^{\star \star \star} p$-value $\leq 0.001$ from a two-tailed, two-sample t-test $(\mathbf{e}, \mathbf{f})$ and Kruskal-Wallis test $(\mathbf{g})$. For expression level is measured by qRT-PCR; three biological replicates and three technical replicates in (c); one biological replicate and three technical replicates for each line in ( $\mathbf{g})$; approximately 20 ears were used in each biological replicate. $n$ is the number of inbred lines examined in $(\mathbf{e}-\mathbf{g})$.

decreased ethylene levels in the aco2-crl line, and increased ethylene levels in the ACO2-OE3 line (Fig. 4h), supporting the idea that $\mathrm{ZmACO} 2$ catalyzes ACC conversion into ethylene in vivo. Together, these results suggest that $Z m A C O 2$ negatively controls kernel number and yield in maize by regulating ethylene biosynthesis. Phytohormones play crucial roles in plant growth, development, and response to various stimuli. The gaseous phytohormone ethylene has long been recognized as a growth inhibitor ${ }^{26,27}$. However, ethylene was found to promote cell division in the quiescent center in the root stem cell niche in Arabidopsis and rice ${ }^{28,29}$. Ethylene also has a dose-dependent effect in opposite directions, both promoting and repressing cell division and fruit elongation in cucumber ${ }^{15}$. Therefore, ethylene appears to have alternating roles, either stimulating or inhibiting growth, depending on endogenous and environmental conditions and developmental stage 26,27 . Here, a higher ethylene level could increase IM size and form more spikelets in developing $q E L 7^{S L 17}$ ears compared to $q E L 7^{Y e 478}$. Usually, IM size is positively correlated with kernel numbers and grain yield in maize $e^{30-33}$. However, sex determination of florets was abnormal in $q E L 7^{S L 17}$ ears, and associated with a reduction in floret fertility, ear length, kernel number, and grain yield, suggesting that floret fertility is also critical for maize grain yield. These findings suggest the role of ethylene in maize grain yield-related traits is more complex than in cucumber fruit size regulation ${ }^{15}$, and the optimization of ethylene levels for grain yield enhancement of maize or other cereal crops needs to balance its effect in both meristem activity and floret fertility.

Ethylene may participate in cross-talk with other phytohormones in developing ears. Recent findings suggest that the function of ethylene in primary root elongation is mediated via interactions with other phytohormones, such as auxin, gibberellin (GA), cytokinins (CK), jasmonic acid (JA), and brassinosteroids $(\mathrm{BR})^{26,27}$. The potential cross-talk between ethylene and other phytohormones could also be observed in transcriptome profiling of developing ears of $q E L 7^{S L 17}$ and $q E L 7^{Y e 478}$ NIL lines, at the $\sim 2 \mathrm{~mm}$ stage where spikelets are being initiated. We first checked the expression of $Z m A C O 2$ homologs in two NIL lines and found another three ZmACO2 homologs expressed $(\mathrm{FPKM}>2)$ in 

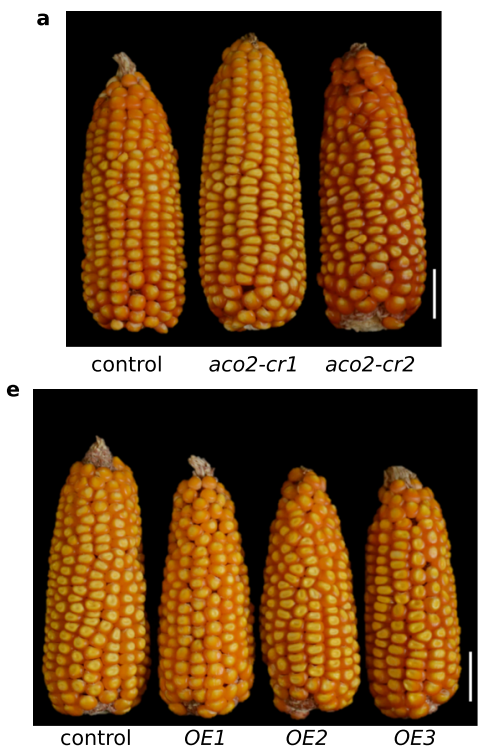
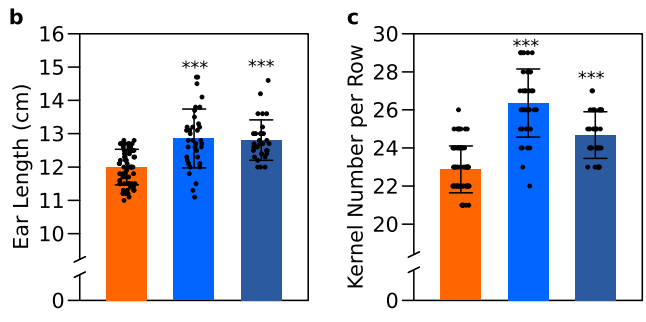

$\square$ control a aco2-cr1 $\square$ aco2-cr2
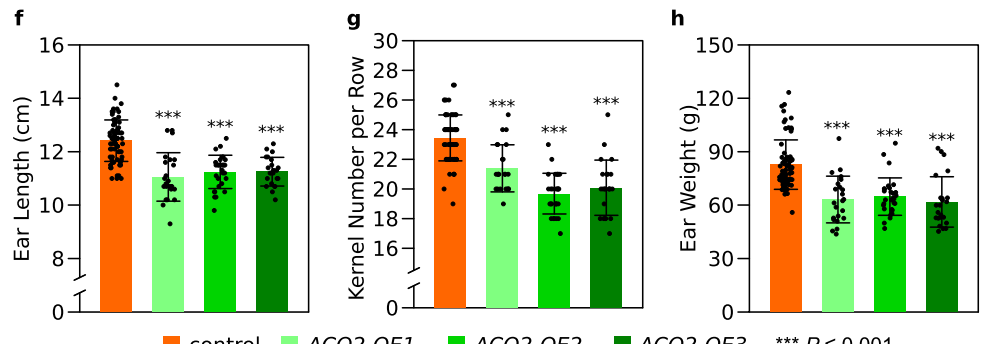

Fig. 3 Validation of ZmACO2 function by coding region knockout and overexpression. a Zmaco2 knockout alleles (aco2-cr1 and aco2-cr2) made longer ears with more kernels than sibling controls; scale bar $=5 \mathrm{~cm}$. Performance of control and Zmaco2 knockout alleles (aco2-cr1 and aco2-cr2) on ear length (b, $p=2.37 \times 10^{-8}$ and $1.87 \times 10^{-9}$ respectively), kernel number per row (c, $p=6.82 \times 10^{-20}$ and $1.44 \times 10^{-9}$ respectively) and ear weight (d, $p=1.41 \times 10^{-5}$ and $1.15 \times 10^{-4}$ respectively); orange bar, control, $n=64$ in (b-d); blue bar, Zmaco2 alleles, $n=36$ ( $\left.a c 02-c r 1\right)$ and 31 (aco2-cr2) in $(\mathbf{b}-\mathbf{d})$, respectively. e ZmACO2 overexpression lines made shorter ears with less kernels than sibling non-transgenic controls; scale bar $=5 \mathrm{~cm}$. $\mathbf{f}-\mathbf{h}$, Performance of ear length $\left(\mathbf{f}, p=5.79 \times 10^{-10}, 1.64 \times 10^{-10}\right.$ and $2.32 \times 10^{-9}$ respectively), kernel number per row $\left(\mathbf{g}, p=3.76 \times 10^{-7}\right.$, $1.99 \times 10^{-19}$ and $2.68 \times 10^{-13}$ respectively) and ear weight (h, $p=5.38 \times 10^{-8}, 1.24 \times 10^{-8}$ and $1.54 \times 10^{-8}$ respectively) in controls and $Z \mathrm{mACO} 2$ overexpression lines; orange bar, control, $n=69$ in ( $\mathbf{f}-\mathbf{h}$ ); green bar, ZmACO2 overexpression line, $n=23$ (ACO2-OE1), 29 (ACO2-OE2) and 23 (ACO2OE3) in $(\mathbf{f}-\mathbf{h})$, respectively. For $(\mathbf{b}-\mathbf{d})$ and $(\mathbf{f}-\mathbf{h})$, data are presented as means \pm SD. ${ }^{\star \star \star} p$-value $\leq 0.001$, from a two-tailed, two-sample t-test. $n$ is the number of ears examined in $(\mathbf{b}-\mathbf{d})$ and $(\mathbf{f}-\mathbf{h})$

developing ears (Fig. 5a, b), suggesting a redundant role of these genes in ethylene biosynthesis. All three genes were downregulated in $q E L 7^{Y e 478}$ similar to $Z m A C O 2$ (Fig. 5a, b), and thus may contribute to the lower ethylene level in the $q E L 7^{Y e 478}$ long ear line.

Ethylene can stimulate auxin biosynthesis, as suggested by previous studies ${ }^{26,27}$. In the low ethylene $q E L 7^{Y e 478}$ line, the expression of seven auxin biosynthesis-related genes were significantly altered, and four of them were downregulated (Fig. 5c and Supplementary Data 6). Notably, another three known auxin-related key regulators of meristem development, $B I F 4^{10}, V T 2^{8}$ and FASCIATED EAR4 (FEA4) ${ }^{34}$, were downregulated in $q E L 7^{Y e 478}$ line (Fig. 5d and Supplementary Data 6). In particular, BIF4, a key component of auxin hormone signaling ${ }^{10}$, was downregulated 5.6 fold, and VT2, which encodes an enzyme in the tryptamine pathway for auxin biosynthesis 8 was down-regulated 1.7 fold. FEA4, which tends to activate genes related to auxin response ${ }^{34}$, was downregulated 2.1 fold. We measured the IAA content in developing ears of the two NIL lines, and found a significant decrease in IAA levels in $q E L 7^{Y e 478}$ (Fig. 5e). Therefore, the auxin biosynthesis and signaling pathways were suppressed in the low ethylene $q E L 7^{Y e 478}$ line, suggesting that ethylene may modulate auxin biosynthesis in developing maize ears.

Most flowering plants produce perfect flowers containing both stamens (male organs) and pistils (female organs) ${ }^{35}$. Although maize florets are initially bisexual, they become unisexual male flowers in the tassel and female flowers in the ear with only stamens or pistils through the process of sex determination ${ }^{5}$. Previous studies suggested JA is necessary for pistil abortion and acquisition of the male characteristics of staminate spikelets ${ }^{36,37}$. In the long ear line $q E L 7^{Y e 478}$, eight JA biosynthesis-related genes were significantly altered, and seven of them were downregulated
(Fig. 5c and Supplementary Data 6). Two known JA biosynthesis genes, Tasselseed 1 (TS1) 36 and Tasselseed $2\left(\right.$ TS2) ${ }^{37}$, were also downregulated 3.0 and 2.3 fold, respectively (Fig. $5 \mathrm{~d}$ and Supplementary Data 6). We measured JA levels in developing ears of the two NIL lines. The long ear $q E L 7^{Y e 478}$ line had a lower level of JA, jasmonyl-isoleucine conjugate (JA-Ile), and JA precursor 12-oxophytodienoic acid (OPDA) (Fig. 5f-i), suggesting a lower JA level may block the conversion to male florets in $q E L 7^{Y e 478}$ ear tips. These results also suggest that ethylene might regulate JA biosynthesis in maize, in contrast to previous reports that JA predominantly acts upstream of ethylene ${ }^{26,27}$. Besides IAA and JA, biosynthesis-related genes of some other phytohormones were also significantly altered, including BR, CK, and GA (Fig. 5c and Supplementary Data 6). These results suggest that ethylene could influence the phytohormone balance in developing maize ears, however, additional studies are needed to support this hypothesis.

Ethylene may regulate inflorescence development-related genes. In addition to phytohormone cross-talk, 22 key genes involved in maize inflorescence development were also significantly altered in $q E L 7^{Y e 478}$ (Fig. 5d and Supplementary Data 6). Of them, six floral meristem determinacy regulators, such as INDETERMINATE SPIKELET1 (IDS1) and TASSELS REPLACE UPPER EARS1 $(T R U 1)^{38,39}$, were downregulated 1.7-3.0 fold (Fig. 5d and Supplementary Data 6). Three RAMOSA-related spikelet determinacy regulators, such as $R A 3$ and $R A 1^{40,41}$, were upregulated 1.6-4.9 fold (Fig. 5d and Supplementary Data 6). Besides, meristem activity regulators, such as $Z m F C P 1$ and $Z m C L E 7^{30-32}$, were either down or upregulated (Fig. 5d and Supplementary Data 6). Therefore, ethylene might affect ear development by phytohormone cross-talk and regulating inflorescence development-related genes, and the underlying mechanisms need to be revealed by further studies. 

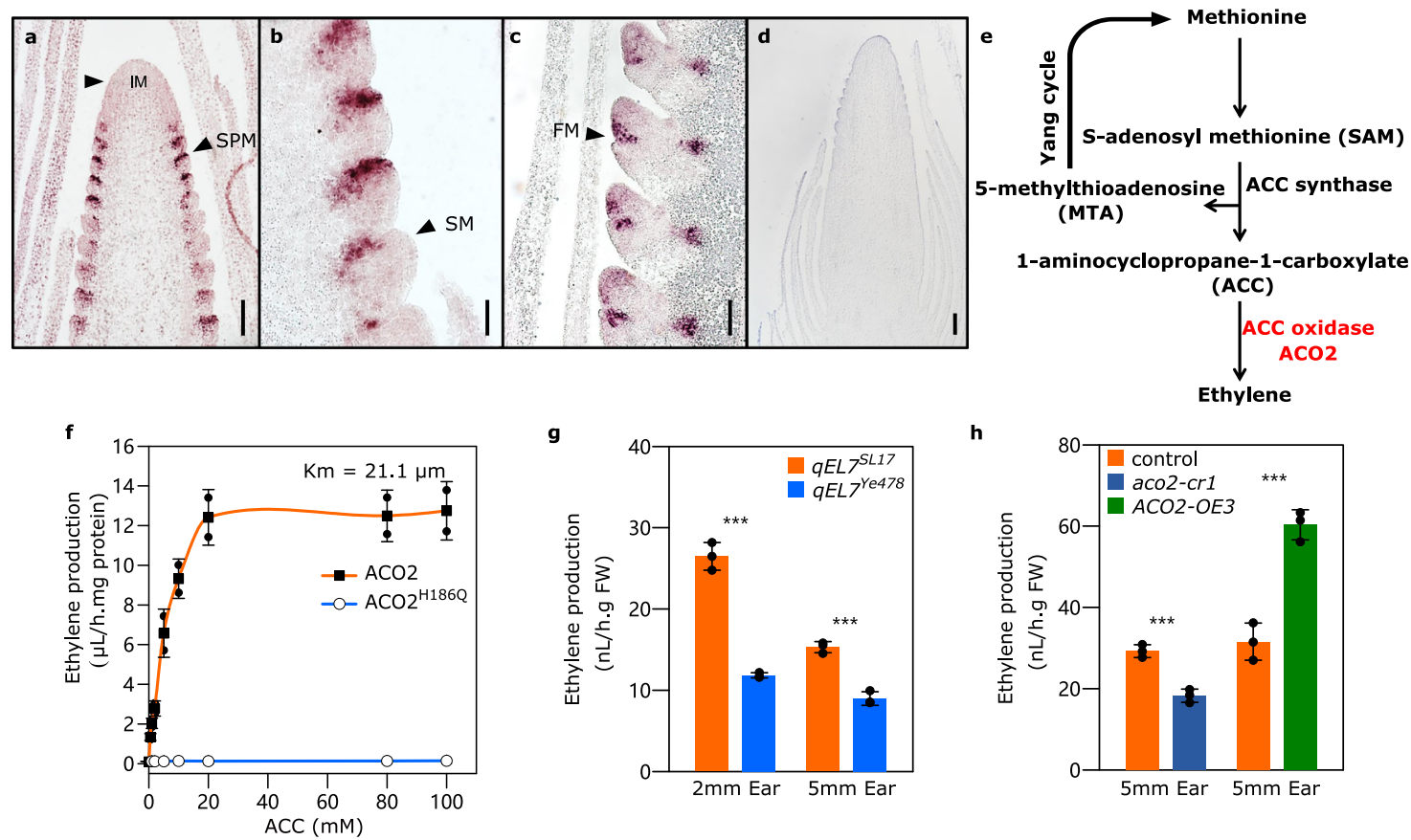

Fig. $4 \mathrm{ZmACO2}$ is expressed in developing ears and shows enzyme activity in ethylene biosynthesis in vivo. ZmACO2 expression is highly localized in developing ears, revealed by RNA in situ hybridization; black arrowheads indicate SPMs (a), SMs (b), and the junction between glumes and FMs (c); the experiment was performed three times with similar results, using ears from at least three independent plants per experiment; negative control using sense probes (d); scale bars $=100 \mu \mathrm{m}$. e ACC oxidase (ACO) converts ACC to ethylene in the final pathway step of methionine-dependent ethylene biosynthesis in plants ${ }^{24}$. f Enzymatic activity of $Z m A C O 2$ and the $\mathrm{H}_{186} \mathrm{Q}$ protein isoforms at various concentrations of $A C C ; \mathrm{H}_{186} \mathrm{Q}$ represents an isoform of ZmACO2 with a substitution at amino acid 186 from a histidine $(\mathrm{H})$ to a glutamine (Q); experimental data are fit to a line representing Michaelis-Menten kinetics. Error bars represent SD; three biological replicates for each. Measurement of endogenous ethylene levels in 2 and $5 \mathrm{~mm}$ ears of $q E L 7$ SL17 (orange bar) and $q E L 7$ Ye478 (blue bar) $(\mathbf{g})\left(p=6.37 \times 10^{-5}\right.$ and $2.67 \times 10^{-4}$ respectively), and $5 \mathrm{~mm}$ ears of aco2-cr7 (dark blue bar) and ACO2-OE3 (dark green bar) and their controls (orange bar) $\left(p=5.50 \times 10^{-4}\right.$ and $5.42 \times 10^{-4}$ respectively). For $\mathbf{g}$, $\mathbf{h}$ data are presented as means \pm SD. ${ }^{\star \star \star} p$-value $\leq 0.001$, from a two-tailed, two-sample t-test, three biological replicates for each, and approximately 30 ears were used in each biological replicate.

The alteration in ZmACO2 expression might also affect ACC content, which is the precursor of ethylene and can act directly to regulate plant growth and development ${ }^{42,43}$. Further evidence is needed to confirm if ACC also regulates maize ear development.

ZmACO2 natural favorable alleles and CRISPR null alleles can enhance grain yield. Grain yield is a complex trait and controlled by numerous QTLs. Some of them have a considerable effect, but only in specific genetic backgrounds, which challenges their application in yield enhancement. Therefore, we evaluated the effect of $q E L 7$ on grain yield-related traits in diverse genetic backgrounds. We first crossed both $q E L 7^{S L 17}$ and $q E L 7^{Y e 478}$ to 13 diverse inbred lines, including eight lines carrying $\mathrm{Hap}^{\mathrm{Ye} 478}$ and five lines carrying $\mathrm{Hap}^{S L 17}$. By comparing to the "inbred $\times$ Hap ${ }^{S L 17 "}$ hybrids, all 13 hybrids derived from "inbred lines $\times$ Hap Ye478" showed longer ears with more kernels and greater ear weight (Supplementary Fig. 5). The ear weight increase ranged from 6.4 to $14.5 \%$, with $10.8 \%$ on average (Supplementary Fig. 5). We also tested the ability of $\mathrm{ZmACO} 2$ to control yield in diverse lines by crossing the aco2-cr1 line, or its wild-type sibling, to six inbred lines; three with $\mathrm{Hap}^{\mathrm{SL} 17}$ and three with Hap Ye478 (Fig. 6a), and made 12 hybrids for yield measurements in three field seasons (Fig. 6b and Supplementary Data 7). We found enhancement of multiple grain yield-related traits in hybrids derived from aco2-crl, including increases in ear length (3.5-7.0\%), kernel number per row $(4.7-6.3 \%)$, ear weight $(9.0-13.9 \%)$, and grain yield per ear $(5.7-21.4 \%)$ relative to hybrids derived from wild-type siblings (Fig. $6 \mathrm{c}-\mathrm{f}$ and Supplementary Data 7). The average increase in grain yield per ear of 12 hybrids was about 13.4\% (Fig. 6f and Supplementary Data 7).
In summary, both the natural $\mathrm{Hap}^{\mathrm{Ye} 478}$ allele and newly created aco2 null CRISPR allele enhance ear length, kernel number, and grain yield under diverse genetic backgrounds.

In summary, we isolated the causal gene $\mathrm{ZmACO} 2$ underlying qEL7. We confirmed that lower ZmACO2 expression resulted in reduced ethylene emission in vivo, but increased the number of fertile florets and kernels, possibly through crosstalk with other hormones and inflorescence developmentrelated genes. The complete or partial loss of $Z m A C O 2$ function through silencing the gene or modifying its promoter can enhance the grain yield of inbred lines and hybrids. Therefore, our findings provide a tool for improving grain yield by optimizing ethylene levels in maize or other cereals and expand our understanding of hormone cross-talk in inflorescence development regulation.

\section{Methods}

Fine mapping of $\boldsymbol{q E L 7}$. We previously detected $q E L 7$ on bin7.02 using a set of $\mathrm{F}_{2.3}$ families derived from a cross of $q E L 7^{S L 17} \times q E L 7^{Y e 478}$. Both $q E L 7^{S L 17}$ and $q E L 7^{Y e 478}$ are a set of near-isogenic lines at $q E L 7$ in the Ye478 genetic background $^{21}$. To fine map $q E L 7$, over $12,000 \mathrm{~F}_{2}$ individuals derived from the $q E L 7^{S L 17} \times q E L 7^{Y e 478}$ were genotyped with eleven markers flanking the $q E L 7$ interval to identify recombinants. The heterozygous recombinant was self-crossed to segregate the homozygous recombinant $(\mathrm{HR})$ and non-recombinant (HNR). The progeny families from HRs and NHRs were phenotyped at Wuhan $\left(30^{\circ} \mathrm{N}, 114^{\circ} \mathrm{E}\right)$ and Ezhou $\left(30^{\circ} \mathrm{N}, 114^{\circ} \mathrm{E}\right)$, China, in 2016 and 2017 under a randomized block design with three replicates. Each plot consisted of 11 individuals grown in a single row with $3 \mathrm{~m}$ in length, spacing of $0.3 \mathrm{~m}$ between plants, and $0.6 \mathrm{~m}$ between rows. Eight to ten competitive individuals were harvested in each plot, and subsequently air-dried to measure the ear length $(\mathrm{cm})$, kernel number per row, kernel row number, ear diameter $(\mathrm{cm})$ and ear weight $(\mathrm{g})$. The sequences of the markers used for fine mapping were listed in Supplementary Data 8. The difference significance was examined using the two-tailed two-sample Student's t-test. 


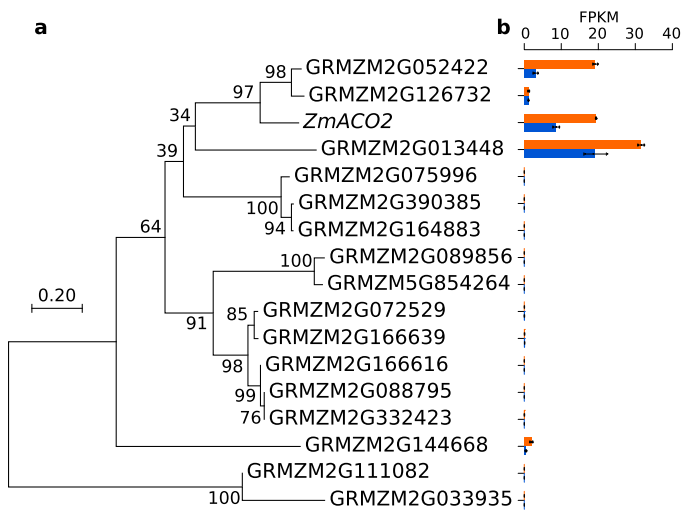

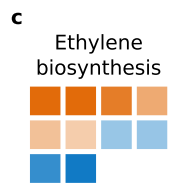

Jasmonic acid biosynthesis

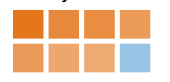

Brassinosteroid biosynthesis
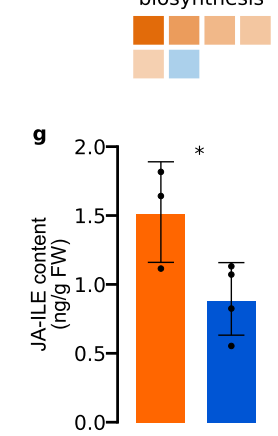
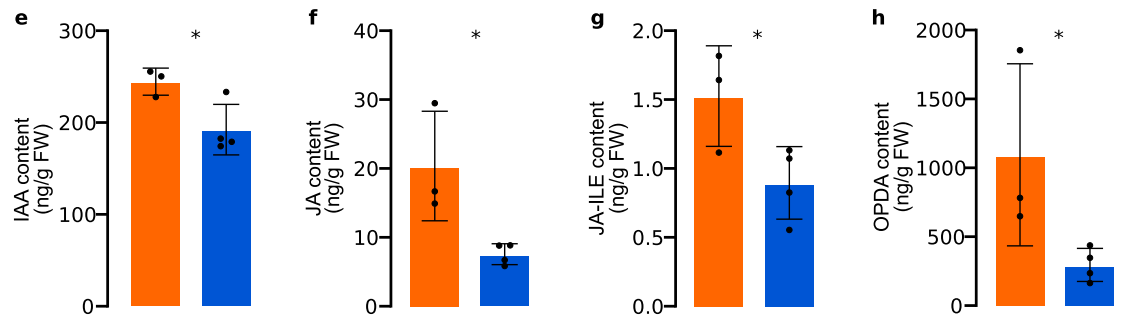

d
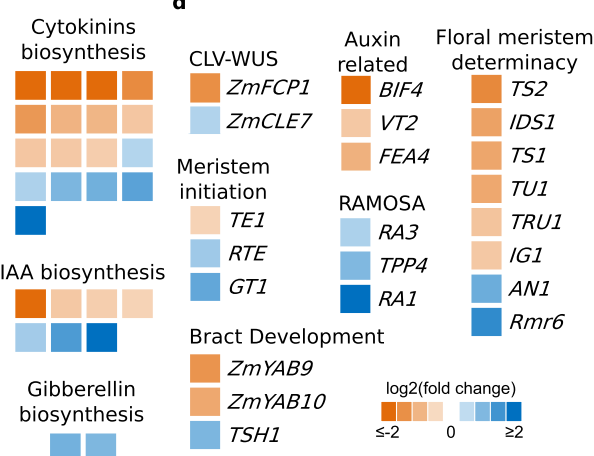

Fig. 5 Expression profiling of developing ears in qEL7 NIL lines revealed that ethylene might cross-talk with other phytohormones and regulate inflorescence development-related genes. Phylogenetic tree (a) of homologous genes of ZmACO2 and their expression levels (b) in developing ears of qEL7 NIL lines; numbers on nodes indicate the bootstrap support of the node (1000 bootstrap replicate percentage); scale bar, branch lengths; FPKM values are shown as mean \pm SD in qEL7SL17 (orange bar) and $q E L 7$ Ye478 (blue bar), three biological replicates for each; Expression changes of phytohormones biosynthesis-related (c) and inflorescence development-related (d) differentially expressed genes (DEGs) of in $q E L 7$ Ye478 relative to qEL7SL17; the colors from orange to blue represent the $\log _{2}(q E L 7 Y e 478 / q E L 7 S L 17)$ from less than -2 to more than 2; the detailed information of gene IDs, annotation, FPKM values, $p$-values and $q$-values of these genes were listed in Supplementary Data 6. $\mathbf{e}-\mathbf{i}$ Phytohormone levels in developing ears of $q E L 7 S L 17$ (orange bar, three biological replicates) and $q E L 7^{Y e 478}$ (blue bar, four biological replicates) including indole-3-acetic acid (IAA, e, $p=0.016$ ), jasmonic acid (JA, $\mathbf{f}, p=0.011$ ), isoleucine conjugate jasmonyl-isoleucine (JA-lle, $\mathbf{g}, p=0.022$ ), and JA precursor 12-oxophytodienoic acid (OPDA, $\mathbf{h}, \mathbf{i} p=0.029$ and 0.025 , respectively); phytohormone levels are given in ng per $\mathrm{g}$ of fresh weight (FW) as mean $\pm \mathrm{SD}$. ${ }^{*} p$-value $\leq 0.05$, from a two-tailed, two-sample t-test; approximately 30 ears were used in each biological replicate in $(\mathbf{e}-\mathbf{i})$.

\section{Analysis of nucleotide diversity and molecular evolution of ZmACO2. A} 3,539 bp region covering $\mathrm{ZmACO} 2$ was resequenced in an association mapping panel with 214 diverse inbred lines by specific primers (Supplementary Data 8), including 1,230 bp upstream 1,635 bp gene body and 674 bp downstream region. Nucleotide diversity was detected by multiple sequence alignment using BioEdit version $7.2 .5^{44}$. Those variants with minor allele frequency (MAF) over $5 \%$ were identified and were then used for an association study. All phenotypes used in this study were measured in Sanya $\left(18^{\circ} \mathrm{N}, 109^{\circ} \mathrm{E}\right)$ in 2019 with two replicates and $\sim$ ten plants for each replicate. Association mapping was performed using a mixed linear model (MLM), considering population structure and relative kinship, in TASSEL version 3.0.67 ${ }^{45-47}$. Pairwise linkage disequilibrium was calculated and then plotted using R software version 3.5.148. A Bonferroni-corrected significance threshold $(p \leq 0.01 / 56=1.79 \mathrm{E}-04)$ was used to identify the significant association. The transcription factor binding site prediction was conducted by PlantPAN 3.0 ${ }^{49}$.

The selection pressure on $\mathrm{ZmACO} 2$ during maize domestication and improvement was estimated in a collection of 44 maize landraces and $53 \mathrm{Z}$. mays subsp. parviglumis teosintes (Supplementary Data 3). The ZmACO2 genomic region was amplified and sequenced using primers listed in Supplementary Data 8. Nucleotide diversity $(\pi)$ and Tajima's D were estimated using DnaSP ver. $5.0^{50}$. Three neutral loci $(a d h 1, a d h 2 \text {, and } t e 1)^{51-53}$ were used as controls for the HKA test $^{54}$ using Zea diploperennis as the outgroup. The overall HKA $P$-value was obtained by summing the individual $\chi^{2}$ values of the three control genes. All of the sequences are listed in source data.

Vector construction, genetic transformation, and identification of the transgenic maize. CRISPR-Cas9 was used to generate null alleles and mutations in the ZmACO2 (Zm00001d020686) promoter region. Single-guide RNAs (sgRNAs) were designed based on the B73 reference genome sequence using the CRISPR-P webtool $^{55}$ (http://cbi.hzau.edu.cn/crispr/). sgRNA arrays were cloned into the CPB binary vector following the manufacturer's suggested protocols ${ }^{56}$. To overexpress $Z m A C O 2$, we cloned a 1,853 bp $Z m A C O 2$ fragment including 1,133 bp coding region and $720 \mathrm{bp} 3^{\prime}$-UTR into the binary vector pZZ01523, which was driven by the maize Ubiquitin (Zm00001d015327) promoter. All plasmids were transformed into inbred line KN5585 via Agrobacterium-mediated transformation ${ }^{57}$ at China National Seed Group Co., Ltd (Wuhan, China). Genomic editing of ZmACO2 was screened by PCR amplification and Sanger sequencing of the target regions. The
Cas 9 negative edited plants were selfed for two generations before further phenotypic scoring and expression analysis. The guide RNA sequences, PCR primers for $Z m A C O 2$, and its promoter genotyping are listed in Supplementary Data 8. Overexpression transgenic lines were confirmed by amplifying the target gene and Ubiquitin promoter with specific primers (Supplementary Data 8).

Gene expression analysis. $Z m A C O 2$ expression was analyzed using developing ears that were collected from B73, two NILs, ZmACO2 promoter-edited lines (ACO2 ${ }^{C R-p r o 1}-A C O 2^{C R}$-pro5), $\mathrm{ZmACO} 2$ overexpression lines (ACO2-OE1-ACO2$O E 3)$, and 40 diverse inbred maize lines, respectively. Total RNA was extracted using TRIzol ${ }^{\oplus}$ Reagent (Life Technologies, Invitrogen, Carlsbad, CA, USA) according to the manufacturer's instructions. Tissue samples were collected from B73 at various developmental stages, including seedling roots, seedling internodes, immature leaves, mature leaves, $2 \mathrm{~mm}$ ears, $5 \mathrm{~mm}$ ears, and $5 \mathrm{~mm}$ tassels. Total RNA of $q E L 7^{S L 17}$ and $q E L 7^{Y e 478}$ was extracted from immature ear stage $1(2 \mathrm{~mm}$ ears at 10-leaf stage with inflorescence meristems (IMs) and spikelet pair meristems (SPMs)), and immature ear stage 2 (5 mm ears at 12-leaf stage with IMs, SPMs, and spikelet-meristems (SMs)). Total RNA of $\mathrm{ZmACO} 2$ promoter edited lines,

ZmACO2 overexpression lines, and 40 diverse maize inbred lines were extracted from immature ears at the S1 stage. DNase I (TaKaRa Biotech, Dalian, China) was used to remove genomic DNA contamination. Oligo (dT) primers and M-MLV reverse transcriptase (Invitrogen, Carlsbad, CA, USA) were used to synthesize firststrand cDNA. Quantitative real-time PCR was performed using Universal SybrGreen Master Mix (Bio-Rad, Hercules, CA, USA) on the CFX96 Real-Time system (Bio-Rad). The maize Actin gene (Zm00001d010159) was used as an internal control. The relative expression of the gene was calculated by the $2^{-\Delta \mathrm{Ct}}$ method. The primers used for quantitative real-time PCR are listed in Supplementary Data 8.

Total RNA of $q E L 77^{S L 17}$ and $q E L 7^{Y e 478}$ from immature ears was sequenced on an Illumina HiSeq 2000 system (Illumina Inc., San Diego, CA USA) in the Beijing Genomics Institute (BGI). Raw RNA-seq reads (on average $\sim 30$ million reads for each) were trimmed with Trimmomatic v. $0.36^{58}$ (parameters:

ILLUMINACLIP:TruSeq3-PE. fa:2:30:10:LEADING:3 TRAILING:3 SLIDINGWINDOW:4:20 MINLEN:50) and then aligned to B73 RefGen v3 reference using TopHat v.2.1.159 (parameters: --b2-sensitive --read-mismatches 2 --read-edit-dist 2 --min-anchor 8 -- splice-mismatches 0 --min-intron-length 50 -- 

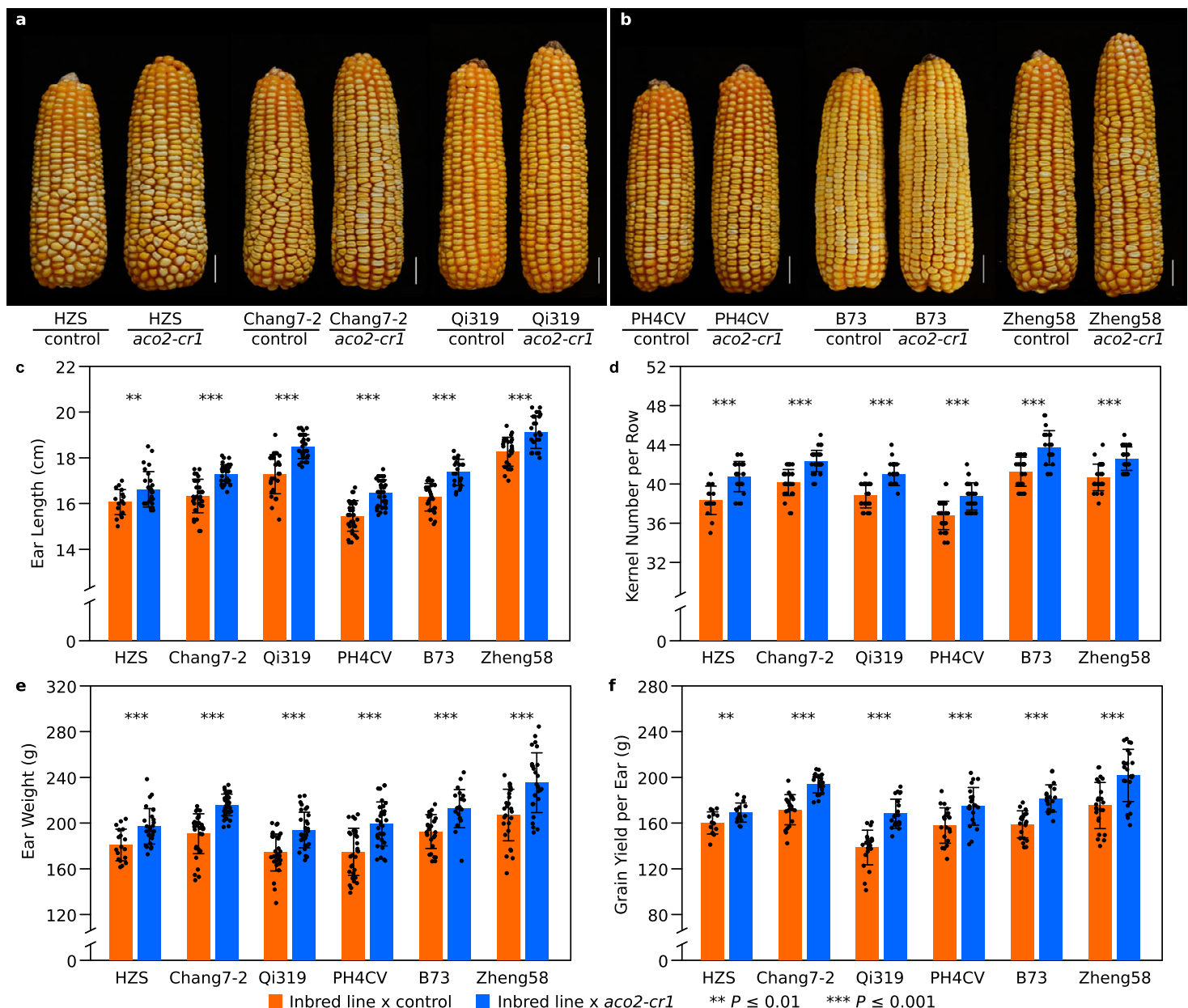

Fig. 6 aco2-cr1 null allele enhances yield-related traits in diverse hybrid backgrounds. Hybrids of "aco2-cr1 $\times$ inbred line" made longer ears with more kernels than "sibling control $\times$ inbred line" hybrids. Diverse inbred lines were crossed to aco2-cr1 and its sibling control; representative mature hybrid ears of aco2-cr1 and its sibling crossed with three Hap SL17 inbred lines (a) and three Hap ${ }^{Y e 478}$ inbred lines (b); scale bar $=5 \mathrm{~cm}$. c-f Performance of hybrids (a, b) in grain ear-traits including ear length $\left(\mathbf{c}, p=0.0053,3.14 \times 10^{-9}, 2.15 \times 10^{-9}, 9.50 \times 10^{-9}, 2.50 \times 10^{-8}\right.$ and $1.33 \times 10^{-5}$ respectively), kernel number per row $\left(\mathbf{d}, p=1.63 \times 10^{-6}, 1.73 \times 10^{-10}, 5.12 \times 10^{-10}, 4.84 \times 10^{-7}, 1.39 \times 10^{-6}\right.$ and $8.59 \times 10^{-7}$ respectively), ear weight $\left(\mathbf{e}, p=3.97 \times 10^{-4}, 1.41 \times 10^{-10}\right.$, $2.82 \times 10^{-6}, 3.71 \times 10^{-6}, 2.18 \times 10^{-5}$ and $4.72 \times 10^{-5}$ respectively $)$ and grain yield per ear $\left(\mathbf{f}, p=0.0054,5.97 \times 10^{-9}, 6.83 \times 10^{-9}, 5.01 \times 10^{-4}, 1.43 \times 10^{-7}\right.$ and $5.69 \times 10^{-5}$ respectively); orange bar, "sibling control $\times$ inbred line", $n=18,34,32,32,28,27$ ears, respectively; blue bar, "aco2-cr $\times$ inbred line", $n=28,34,32,32,22,26$ ears, respectively. Data are presented as means \pm SD. ${ }^{\star \star} p$-value $\leq 0.01$, ${ }^{\star \star \star} p$-value $\leq 0.001$, from a two-tailed, two-sample t-test.

max-intron-length 50,000 -- max-multihits 20). Next, Cufflinks v2.2.160 was used to calculate the gene expression level and call differentially expressed gene $(p<0.05$, $q<0.05$ and fold change $>1.5)$. The maize phytohormones biosynthesis-related genes were annotated by MaizeCyc v2.261.

Immature B73 ears ( $5 \mathrm{~mm}$ ) were freshly collected and fixed in $4 \%$ paraformaldehyde (Electron Microscopy Sciences, $15714 \mathrm{~s}$ ) for $16 \mathrm{~h}$ at $4{ }^{\circ} \mathrm{C}$, and dehydrated through a graded alcohol series $(50,70,85,95$, and $100 \%)$ and a histoclear series (National diagnostics, HS-202), then embedded in paraplast (McCormick Scientific, 39503002). 8-10 $\mu \mathrm{m}$ sections were cut using a Leica microtome (Leica RM2265), then mounted on ProbeOn Plus Slides (Fisher Scientific, 22-230-900). The probe fragment was amplified from B73 immature ears cDNA using primers ACO2-F (5'-ATGGCGCCTGCATTGTCATT-3') and ACO2R (5'-TCACGCGATGGCTATGCGAT- $3^{\prime}$ ), and subcloned into pEASY-T1 (TransGen Biotech, CT101-01). Clones carrying an insertion in both orientations were identified and sequence-verified to generate antisense or sense probes by in vitro transcription with T7 polymerase (Sigma-Aldrich, 10881775001) and NTP cocktail. The NTP cocktail contains ribonucleoside triphosphate set (Roche, 11277057001) including ATP, GTP, CTP and 1:1 molar ratio of digoxigenin-11uridine triphosphate (DIG-11-UTP, Roche, 11209256910): cold (unlabeled) UTP nucleotide (Roche, 11277057001). Hybridization was performed according to Jackson (1994) ${ }^{62}$ and probes were then applied on tissue sections and incubated at $50{ }^{\circ} \mathrm{C}$ overnight. After hybridization, the sections were washed in $0.2 \times$ SSC and treated by RNaseA (Roche, 10109142001). Then the sections were incubated with anti-digoxigenin antibody (Anti-Digoxigenin-AP Fab fragments, Roche, 11093274910 ) at a concentration of 1:1250 for two hours at room temperature and washed in 1\% Bovine Serum Albumin buffer (Sigma, B2064-50G). Sections were incubated with freshly dissolved NBT/BCIP (NBT/BCIP Ready-to-Use Tablets,
Roche, 11697471001) solution for $12-15 \mathrm{~h}$ before mounting, and imaged using a Nikon DS-Ri2 DIC microscope.

In vitro ACO activity assays. The $Z m A C O 2$ coding sequence was cloned into pET28a-sumo (Novagene), and site-directed mutagenesis was performed to introduce the point mutation $\mathrm{H}_{186} \mathrm{Q}$ described in the results section. Both sequences were verified by Sanger sequencing before transformation into the Rosetta E. coli strain. Cultures were grown to an $\mathrm{OD}_{600}$ of 0.6 at $37^{\circ} \mathrm{C}$, cooled to $16^{\circ} \mathrm{C}$ prior to addition of isopropyl $\beta$-D-1-thiogalactopyranoside to a final concentration of $0.5 \mathrm{mM}$, and grown for an additional $12-16 \mathrm{~h}$ at $16^{\circ} \mathrm{C}$. Purification of His-ZmACO2 was performed with the Ni-NTA protein purification system (QIAGEN) according to the manufacturer's instructions. Protein purity and concentration were assessed using SDS-PAGE gels.

The ZmACO2 activity assay was carried out in a scintillation vial (VWR Collection, VWRU66022-065) ${ }^{24}$. The standard reaction of a $5 \mathrm{~mL}$ mixture in a sealed $10 \mathrm{~mL}$ test vial contained $50 \mathrm{mM} 4$-morpholinepropanesulfonic acid (pH 7.2), $5 \mathrm{mM}$ ascorbic acid sodium salt, $20 \mathrm{mM}$ sodium bicarbonate, $0.02 \mathrm{mM}$ iron sulfate, $1 \mathrm{mM}$ ACC, $1 \mathrm{mM}$ dithiothreitol, and $10 \%$ glycerol (v/v.) The reaction was initiated by adding $1 \mu \mathrm{g}$ of purified recombinant enzyme. After incubation for $1 \mathrm{~h}$ at $30^{\circ} \mathrm{C}$ with shaking, $1 \mathrm{~mL}$ of gas was withdrawn with a syringe from the headspace of the sealed $10 \mathrm{~mL}$ tube for ethylene quantification using an Agilent 7890B gas chromatograph fitted with a flame-ionization detector. The standard curve was created with standard concentrations of ethylene: $1,2,3$, and $5 \mu \mathrm{L} / \mathrm{L}$, with the equation $y=12.51 x-0.914 ; r^{2}=0.998$ ( $y$ : peak area of ethylene; $x$ : the concentration of ethylene in the sample; $r^{2}$ : coefficient of determination). The results shown are the mean of three replicates. 
Endogenous ethylene measurement. To measure endogenous ethylene production, we collected immature ears from the two NILs (2 and $5 \mathrm{~mm}$ ) and transgenic lines $(5 \mathrm{~mm})$, respectively. Samples were placed in a $10 \mathrm{~mL}$ container with $2 \mathrm{~mL} \mathrm{LB}$ medium and sealed with a rubber stopper. After incubation at $25^{\circ} \mathrm{C}$ for $24 \mathrm{~h}$ in the dark, $1 \mathrm{~mL}$ of gas was withdrawn using a gas-tight syringe from the headspace and injected into a gas chromatograph (Agilent 7890B, USA) equipped with a flameionization detector and a capillary column for ethylene measurement. The ethylene production rate (microliters per gram fresh weight per hour) was calculated on the basis of the initial fresh weight of the immature ears. 30-50 plants were used for each biological replicate, and three biological replicates for each. The difference significance was calculated using a two-tailed Student's t-test.

Measurement of endogenous IAA and JA levels. We collected immature ears $(\sim 0.5-1 \mathrm{~cm})$ from $q E L 7^{S L 17}$ and $q E L 7^{Y e 478}$ with at least three biological replicates to measure endogenous hormone levels. Each sample was weighed and ground to powder in liquid nitrogen. Approximately $100 \mathrm{mg}$ powder was added to $1 \mathrm{~mL}$ of 1propanol/water/concentrated $\mathrm{HCl}(2 / 1 / 0.002$, v/v/v) and vortexed for $30 \mathrm{~min}$ at $4{ }^{\circ} \mathrm{C}$. Then $1 \mathrm{~mL}$ methylene chloride $\left(\mathrm{CH}_{2} \mathrm{Cl}_{2}\right)$ was added and vortexed for $30 \mathrm{~min}$ at $4{ }^{\circ} \mathrm{C}$. After centrifugation at $13,000 \times g$ for $10 \mathrm{~min}$ at $4{ }^{\circ} \mathrm{C}$, the lower layer (around $1 \mathrm{~mL}$ ) was transferred to a new tube and dried under a stream of nitrogen. The dried samples were dissolved in $200 \mu \mathrm{L}$ of methanol/formic acid (1/1, v/v), filtered through a $0.45 \mu \mathrm{m}$ membrane, and transferred to sample vials for analysis in a HPLC-MS/MS system (QTRAP ${ }^{\circledast} 6500+$ LC-MS/MS System, SCIEX) consisting of HPLC and QTRAP 6500 system. Indole-3-acetic acid (IAA) and jasmonic acid (JA), including isoleucine conjugate jasmonyl-isoleucine (JA-Ile), JA precursor 12-oxophytodienoic acid (OPDA) 63,64, were measured at National Key Laboratory of Crop Genetic Improvement, Hubei Hongshan Laboratory, Huazhong Agricultural University (Wuhan, China). The contents of IAA and JA were determined by comparison of the response to the internal standards (mol) added during extraction to the ear sample components. The hormone loss during sample preparation and chromatography was corrected by the addition of internal standards during extraction, then combined with the weight of the sample to calculate ng/g fresh weight ${ }^{65}$

Evaluation of grain yield-related traits. The phenotypes of $Z m A C O 2$ knockout lines, $\mathrm{ZmACO} 2$ promoter edited lines, $\mathrm{ZmACO} 2$ overexpression lines, and their corresponding wide-type sibling controls were investigated at Ezhou $\left(30^{\circ} \mathrm{N}, 114^{\circ} \mathrm{E}\right)$ or Sanya $\left(18^{\circ} \mathrm{N}, 109^{\circ} \mathrm{E}\right)$ in 2019 , and Sanya $\left(18^{\circ} \mathrm{N}, 109^{\circ} \mathrm{E}\right)$ in 2020 . To evaluate alleles in different genetic backgrounds, a total of 13 lines, including $5 \mathrm{Hap}^{\mathrm{SL} 17}$ lines and $8 \mathrm{Hap}{ }^{Y e 478}$ lines, were crossed to both $q E L 7^{S L 17}$ and $q E L 7^{Y e 478}$, respectively. The 26 hybrids were grown at Ezhou $\left(30^{\circ} \mathrm{N}, 114^{\circ} \mathrm{E}\right)$ in 2018 spring. We also separately crossed the aco2-crl line and its wild-type sibling to six inbred lines comprised of three Hap ${ }^{S L 17}$ lines and three Hap ${ }^{Y e 478}$ lines. The 12 hybrids were grown at Ezhou $\left(30^{\circ} \mathrm{N}, 114^{\circ} \mathrm{E}\right)$ in 2019 , Xiangyang $\left(30^{\circ} \mathrm{N}, 112^{\circ} \mathrm{E}\right)$ and Zhangye $\left(39^{\circ} \mathrm{N}, 100^{\circ} \mathrm{E}\right)$ in 2020 . The field experiment followed a randomized block design with three replicates. Each plot consisted of 11 individuals grown in a single row of $3 \mathrm{~m}$ in length, spacing of $0.3 \mathrm{~m}$ between plants, and $0.6 \mathrm{~m}$ between rows. Eight to ten competitive individuals were harvested in each plot, and subsequently air-dried to measure the ear length $(\mathrm{cm})$, kernel number per row and ear weight $(\mathrm{g})$, and grain yield per ear $(\mathrm{g})$. The difference significance was calculated using a two-tailed, two-sample Student's t-test.

Reporting summary. Further information on research design is available in the Nature Research Reporting Summary linked to this article.

\section{Data availability}

Source data are available. The genetic materials that support the findings of this study and raw data used for hormone quantification are available from the corresponding authors upon request. The RNA-seq datasets are available from the National Center for Biotechnology Information, the BioProject and SRA accession numbers are [https:// www.ncbi.nlm.nih.gov/bioproject/PRJNA705033] and SRR13787130

SRR13787135. Source data are provided with this paper.

Received: 6 April 2021; Accepted: 14 September 2021; Published online: 05 October 2021

\section{References}

1. Godfray, H. C. et al. Food security: the challenge of feeding 9 billion people. Science 327, 812c18 (2010).

2. Ray, D. K., Mueller, N. D., West, P. C. \& Foley, J. A. Yield trends are insufficient to double global crop production by 2050. PLoS One 8, e66428 (2013).

3. Lopez-Reynoso, J. J. \& Hallauer, A. R. Twenty-seven cycles of divergent mass selection for ear length in maize. Crop Sci. 38, 1099-1107 (1998).
4. Cheng, P., Greyson, R. \& Walden, D. Organ initiation and the development of unisexual flowers in the tassel and ear of Zea mays. Am. J. Bot. 70, 450-462 (1983).

5. Vollbrecht, E. \& Schmidt, R. J. In Handbook of Maize: Its Biology 13-40 (Springer, 2009).

6. Wu, Q., Xu, F. \& Jackson, D. All together now, a magical mystery tour of the maize shoot meristem. Curr. Opin. Plant Biol. 45, 26-35 (2018).

7. Gallavotti, A. et al. Sparse inflorescencel encodes a monocot-specific YUCCAlike gene required for vegetative and reproductive development in maize. Proc. Natl Acad. Sci. USA 105, 15196-15201 (2008).

8. Phillips, K. A. et al. Vanishing tassel2 encodes a grass-specific tryptophan aminotransferase required for vegetative and reproductive development in maize. Plant Cell 23, 550-566 (2011).

9. Mcsteen, P. et al. Barren inflorescence2 encodes a co-ortholog of the PINOID serine/threonine kinase and is required for organogenesis during inflorescence and vegetative development in maize. Plant Physiol. 144, 1000-1011 (2007).

10. Galli, M. et al. Auxin signaling modules regulate maize inflorescence architecture. Proc. Natl Acad. Sci. USA 112, 13372-13377 (2015).

11. Lin, Z. et al. A tomato HB-ZIP homeobox protein, LeHB-1, plays an important role in floral organogenesis and ripening. Plant J. 55, 301-310 (2008).

12. Martinis, D. \& Mariani, C. Silencing gene expression of the ethylene-forming enzyme results in a reversible inhibition of ovule development in transgenic tobacco plants. Plant Cell 11, 1061-1072 (1999).

13. Martínez, C. et al. Involvement of ethylene biosynthesis and signalling in fruit set and early fruit development in zucchini squash (Cucurbita pepo L.). BMC Plant Biol. 13, 139 (2013).

14. Chen, H. et al. An ACC oxidase gene essential for cucumber carpel development. Mol. Plant 9, 1315-1327 (2016).

15. Xin, T. et al. Genetic regulation of ethylene dosage for cucumber fruit elongation. Plant Cell 31, 1063-1076 (2019).

16. Cox, W. J. \& Andrade, H. F. Growth, yield, and yield components of maize as influenced by ethephon. Crop Sci. 28, 536-542 (1988).

17. Langan, T. D. \& Oplinger, E. S. Growth and yield of ethephon treated maize Agron. J. 79, 130-134 (1987).

18. Habben, J. E. et al. Transgenic alteration of ethylene biosynthesis increases grain yield in maize under field drought-stress conditions. Plant Biotechnol. J. 12, 685-693 (2014)

19. Shi, J. et al. ARGOS8 variants generated by CRISPR-Cas9 improve maize grain yield under field drought stress conditions. Plant Biotechnol. J. 15, 207-216 (2017).

20. Shi, J. et al. Overexpression of ARGOS genes modifies plant sensitivity to ethylene, leading to improved drought tolerance in both Arabidopsis and maize. Plant Physiol. 169, 266-282 (2015).

21. Zhou, G. et al. qEL7.2 is a pleiotropic QTL for kernel number per row, ear length, and ear weight in maize (Zea mays L.). Euphytica 203, 429-436 (2015).

22. Sun, Y. et al. 3D genome architecture coordinates trans and cis regulation of differentially expressed ear and tassel genes in maize. Genome Biol. 21, 143 (2020).

23. Parvathaneni, R. K. et al. The regulatory landscape of early maize inflorescence development. Genome Biol. 21, 165 (2020).

24. Bulens, I. et al. Protocol: an updated integrated methodology for analysis of metabolites and enzyme activities of ethylene biosynthesis. Plant Methods 7, 17 (2011).

25. Zhang, Z., Ren, J. S., Clifton, I. J. \& Schofield, C. J. Crystal structure and mechanistic implications of 1-aminocyclopropane-1-carboxylic acid oxidasethe ethylene-forming enzyme. Chem. Biol. 11, 1383-1394 (2004).

26. Van de Poel, B., Smet, D. \& Van Der Straeten, D. Ethylene and hormonal cross talk in vegetative growth and development. Plant Physiol. 169, 61-72 (2015).

27. Qin, H., He, L. \& Huang, R. The coordination of ethylene and other hormones in primary root development. Front. Plant Sci. 10, 874 (2019).

28. Ortega-Martínez, O., Pernas, M., Carol, R. J. \& Dolan, L. Ethylene modulates stem cell division in the Arabidopsis thaliana root. Science 317, 507-510 (2007).

29. Ni, J., Shen, Y., Zhang, Y. \& Wu, P. Definition and stabilisation of the quiescent Centre in rice roots. Plant Biol. 16, 1014-1019 (2014).

30. Rodríguez-Leal, D. et al. Evolution of buffering in a genetic circuit controlling plant stem cell proliferation. Nat. Genet. 51, 786-792 (2019).

31. Liu, L. et al. Enhancing grain-yield-related traits by CRISPR-Cas9 promoter editing of maize CLE genes. Nat. Plants 7, 287-294 (2021).

32. Je, B. I. et al. Signaling from maize organ primordia via FASCIATED EAR3 regulates stem cell proliferation and yield traits. Nat. Genet. 48, 785-791 (2016).

33. Bommert, P., Nagasawa, N. S. \& Jackson, D. Quantitative variation in maize kernel row number is controlled by the FASCIATED EAR2 locus. Nat. Genet. 45, 334-337 (2013).

34. Pautler, M. et al. FASCIATED EAR4 encodes a bZIP transcription factor that regulates shoot meristem size in maize. Plant Cell 27, 104-120 (2015). 
35. Irish, E. E. Regulation of sex determination in maize. Bioessays 18, 363-369 (1996).

36. Acosta, I. F. et al. tasselseed 1 is a lipoxygenase affecting jasmonic acid signaling in sex determination of maize. Science 323, 262-265 (2009).

37. DeLong, A., Calderon-Urrea, A. \& Dellaporta, S. L. Sex determination gene TASSELSEED2 of maize encodes a short-chain alcohol dehydrogenase required for stage-specific floral organ abortion. Cell 74, 757-768 (1993).

38. Chuck, G., Meeley, R. \& Hake, S. Floral meristem initiation and meristem cell fate are regulated by the maize AP2 genes ids1 and sid1. Development 135, 3013-3019 (2008).

39. Dong, Z. et al. Ideal crop plant architecture is mediated by tassels replace upper ears1, a BTB/POZ ankyrin repeat gene directly targeted by TEOSINTE BRANCHED1. Proc. Natl Acad. Sci. USA 114, E8656 LP-E8658664 (2017).

40. Satoh-Nagasawa, N., Nagasawa, N., Malcomber, S., Sakai, H. \& Jackson, D. A trehalose metabolic enzyme controls inflorescence architecture in maize. Nature 441, 227-230 (2006).

41. Gallavotti, A. et al. The control of axillary meristem fate in the maize ramosa pathway. Development 137, 2849-2856 (2010).

42. Mou, W. et al. Ethylene-independent signaling by the ethylene precursor ACC in Arabidopsis ovular pollen tube attraction. Nat. Commun. 11, 4082 (2020).

43. Li, D. et al. Ethylene-independent functions of the ethylene precursor ACC in Marchantia polymorpha. Nat. Plants 6, 1335-1344 (2020).

44. Hall, T. A. BioEdit: a user-friendly biological sequence alignment editor and analysis program for Windows 95/98/NT. Nucleic Acids Symp . Ser. 41, 95-98 (1999).

45. Yu, J. et al. A unified mixed-model method for association mapping that accounts for multiple levels of relatedness. Nat. Genet. 38, 203-208 (2006).

46. Yang, Q. et al. CACTA-like transposable element in ZmCCT attenuated photoperiod sensitivity and accelerated the post-domestication spread of maize. Proc. Natl Acad. Sci. USA 110, 16969-16974 (2013).

47. Bradbury, P. J. et al. TASSEL: software for association mapping of complex traits in diverse samples. Bioinformatics 23, 2633-2635 (2007).

48. Shin, J. H., Blay, S., McNeney, B. \& Graham, J. LD heatmap: an R function for graphical display of pairwise linkage disequilibria between single nucleotide polymorphisms. J. Stat. Softw. 16, 1-10 (2006).

49. Chow, C. N. et al. PlantPAN3.0: a new and updated resource for reconstructing transcriptional regulatory networks from ChIP-seq experiments in plants. Nucleic Acids Res. 47, D1155-D1163 (2019).

50. Librado, P. \& Rozas, J. DnaSP v5: a software for comprehensive analysis of DNA polymorphism data. Bioinformatics 25, 1451-1452 (2009).

51. Eyre-Walker, A., Gaut, R. L., Hilton, H., Feldman, D. L. \& Gaut, B. S. Investigation of the bottleneck leading to the domestication of maize. Proc. Natl Acad. Sci. USA 95, 4441-4446 (1998).

52. Tenaillon, M. I. et al. Patterns of DNA sequence polymorphism along chromosome 1 of maize (Zea mays ssp. mays L.). Proc. Natl Acad. Sci. USA 98, 9161-9166 (2001).

53. White, S. E. \& Doebley, J. F. The molecular evolution of terminal ear1, a regulatory gene in the genus Zea. Genetics 153, 1455-1462 (1999).

54. Hudson, R. R., Kreitman, M. \& Aguadé, M. A test of neutral molecular evolution based on nucleotide data. Genetics 116, 153-159 (1987).

55. Lei, Y. et al. CRISPR-P: a web tool for synthetic single-guide RNA design of CRISPR-system in plants. Mol. Plant 7, 1494-1496 (2014).

56. $\mathrm{Li}$, C. et al. RNA-guided Cas9 as an in vivo desired-target mutator in maize. Plant Biotechnol. J. 15, 1566-1576 (2017).

57. Ishida, Y., Hiei, Y. \& Komari, T. Agrobacterium-mediated transformation of maize. Nat. Protoc. 2, 1614-1621 (2007).

58. Bolger, A. M., Lohse, M. \& Usadel, B. Trimmomatic: a flexible trimmer for Illumina sequence data. Bioinformatics 30, 2114-2120 (2014).

59. Kim, D. et al. TopHat2: accurate alignment of transcriptomes in the presence of insertions, deletions, and gene fusions. Genome Biol. 14, R36 (2013).

60. Trapnell, C. et al. Transcript assembly and quantification by RNA-Seq reveals unannotated transcripts and isoform switching during cell differentiation. Nat. Biotechnol. 28, 511-515 (2010).

61. Monaco, M. K. et al. Maize metabolic network construction and transcriptome analysis. Plant Genome 6, 1-12 (2013).

62. Jackson, D., Veit, B. \& Hake, S. Expression of maize KNOTTED1 related homeobox genes in the shoot apical meristem predicts patterns of morphogenesis in the vegetative shoot. Development 120, 405-413 (1994).
63. Wasternack, C. \& Hause, B. The missing link in jasmonic acid biosynthesis. Nat. Plants 5, 776-777 (2019).

64. Wasternack, C. \& Feussner, I. The oxylipin pathways: biochemistry and function. Annu. Rev. Plant Biol. 69, 363-386 (2018).

65. Pan, X., Welti, R. \& Wang, X. Simultaneous quantification of major phytohormones and related compounds in crude plant extracts by liquid chromatography-electrospray tandem mass spectrometry. Phytochemistry 69 1773-1781 (2008)

\section{Acknowledgements}

This work was supported by the National Key Research and Development Program of China (2016YFD0100404), the National Natural Science Foundation of China (91935305, 31871628), the National Science Foundation (IOS-1546837 and 2129189 to D.J.), and Office of China Postdoctoral Affairs Fellowship (Fellowship to L.L.). We are grateful to Prof. Ping Yin (National Key Laboratory of Crop Genetic Improvement, Huazhong Agricultural University) for his help with protein expression and purification, and are also grateful to Xingrong Wang and Yongsheng Li (Institute of Crop Sciences, Gansu Academy of Agricultural Sciences) for their assistance in phenotype evaluation. We are also grateful to Penelope Lindsay (Cold Spring Harbor Laboratory) for help in paper editing.

\section{Author contributions}

Z.Z., L.L. and D.J. conceived and designed the experiments. Q.N., Y.D., Y.L., X.S., H.J. R.Z., and J.Z. performed the QTL mapping. Q.N., L.L., and H.J. performed the association mapping. Q.N., Y.J., and F.Y. analyzed the gene expression. Y.J. designed the CRISPR sgRNAs and prepared the constructs for maize transformation. Q.N. performed $\mathrm{ZmACO} 2$ function analysis and transcriptome analysis. L.L. prepared the figures and together with Q.N. and Y.J. L.L. wrote the paper. All authors read and approved the paper.

\section{Competing interests}

The authors declare no competing interests.

\section{Additional information}

Supplementary information The online version contains supplementary material available at https://doi.org/10.1038/s41467-021-26123-z.

Correspondence and requests for materials should be addressed to David Jackson, Lei Liu or Zuxin Zhang.

Peer review information Nature Communications thanks Bram Van de Poel and Feng Tian for their contribution to the peer review of this work. Peer reviewer reports are available.

Reprints and permission information is available at http://www.nature.com/reprints

Publisher's note Springer Nature remains neutral with regard to jurisdictional claims in published maps and institutional affiliations.

Open Access This article is licensed under a Creative Commons Attribution 4.0 International License, which permits use, sharing, adaptation, distribution and reproduction in any medium or format, as long as you give appropriate credit to the original author(s) and the source, provide a link to the Creative Commons license, and indicate if changes were made. The images or other third party material in this article are included in the article's Creative Commons license, unless indicated otherwise in a credit line to the material. If material is not included in the article's Creative Commons license and your intended use is not permitted by statutory regulation or exceeds the permitted use, you will need to obtain permission directly from the copyright holder. To view a copy of this license, visit http://creativecommons.org/ licenses/by/4.0\%

(C) The Author(s) 2021 